\title{
Peripheral vascular disease: shaping a new panvascular interventional approach
}

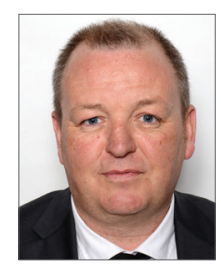

Paul Cummins, Editorial Manager; Patrick W. Serruys, Editor-in-Chief

Whereas for many years our profession's sole remit was the focus on balloon angioplasty and later stents for coronary interventions, more recently our attention moved on, turning as well to valvular interventions. Transcatheter aortic value implantations and the very recent developments in transcatheter mitral value implantations are of course specialties that captivate increasing larger audiences: a prime example is the record attendance of 2,297 active participants at PCR London Valves this year in Berlin. The fields of non-valvular structural interventions and interventions for hypertension and heart failure are also witnessing an increasing academic enthusiasm and exploration from the interventionalist. Comparing the skill sets and competences of the interventionalist today with those of his colleague of 20 or even 10 years ago is quite simply an exercise in illumination and amazement. Yet, as we carry on building our professional knowledge base, well rounded as it is now, there is still more to pander to our intellectual curiosity.

One could now argue that it now seems as if peripheral vascular disease is the next frontier of interest for the interventionalist. Philosophising on this topic, one quickly recognises that vascular medicine is not confined to interventional therapy and medical care of the vascular patient alone. It is imperative that one understands the aetiology, pathophysiology and natural history of vascular disease, as well as possessing a robust grasp of medical, surgical and interventional technologies. After all, the interventionalist understands that atherosclerosis is not primarily located in the coronary vasculature but also exists within the peripheral vasculature.

Patients with peripheral vascular disease generally present with a similar risk factor profile to the cardiovascular patient and thus by definition share the same preventive measures, and undergo intrinsically comparable therapeutic management strategies based on the universal fundamentals of revascularisation.
A recent meta-analysis in The Lancet reported that the prevalence of peripheral artery disease worldwide has risen by $23.5 \%$ in the past 10 years to 202 million in $2010^{1}$. At first sight, this was an alarming statistic; however, the accompanying editorialist declared that the results were in fact actually underestimating the true burden of peripheral artery disease, as the estimates were derived from the use of the ankle-brachial index. Disturbingly, the editorial concluded, "when any disease affects more than 200 million people, it is time to take action to prevent and control its global burden"2.

To help address this global burden, for the inaugural PCR Istanbul Peripheral Course later this month, Alberto Cremonesi, Ömer Goktekin and Thomas Zeller, as course directors, have issued a call for "a new holistic vision for cardiologists: panvascular interventional medicine". They envisage a future where, by answering this call and by "overcoming interdisciplinary boundaries and building effective horizontal integration", the whole gamut of cardiovascular diseases can be tackled by the cardiological interventionalist.

And, as our professional knowledge base grows, we should remember that Plato, not only a philosopher and mathematician but also the founder of the Academy in Athens, the first institution of higher learning in the Western world, told us, "a good decision is based on knowledge and not on numbers".

\section{References}

1. Fowkes FG, Rudan D, Rudan I, Aboyans V, Denenberg JO, McDermott MM, Norman PE, Sampson UK, Williams LJ, Mensah GA, Criqui MH. Comparison of global estimates of prevalence and risk factors for peripheral artery disease in 2000 and 2010: a systematic review and analysis. Lancet. 2013;382: 1329-40.

2. Hirsch AT, Duval S. The global pandemic of peripheral artery disease. Lancet. 2013;382:1312-4. 